\title{
Spatial Heterogeneities in Structural Temperature Cause Kovacs' Expansion Gap Paradox in Aging of Glasses
}

\author{
Matteo Lulli®, ${ }^{1,2}$ Chun-Shing Lee, ${ }^{1}$ Hai-Yao Deng $\odot,{ }^{3,4}$ Cho-Tung Yip $\odot,{ }^{5}$ and Chi-Hang Lam ${ }^{1, *}$ \\ ${ }^{1}$ Department of Applied Physics, Hong Kong Polytechnic University, Hong Kong, China \\ ${ }^{2}$ Department of Mechanics and Aerospace Engineering, Southern University of Science and Technology, \\ Shenzhen, Guangdong 518055, China \\ ${ }^{3}$ School of Physics and Astronomy, The University of Manchester, Manchester M13 9PL, United Kingdom \\ ${ }^{4}$ School of Physics and Astronomy, Cardiff University, The Parade, Cardiff CF24 3AA, United Kingdom \\ ${ }^{5}$ School of Science, Harbin Institute of Technology, Shenzhen Graduate School, Shenzhen, Guangdong 518055, China
}

(Received 6 September 2019; accepted 5 February 2020; published 6 March 2020)

\begin{abstract}
Volume and enthalpy relaxation of glasses after a sudden temperature change has been extensively studied since Kovacs' seminal work. One observes an asymmetric approach to equilibrium upon cooling versus heating and, more counterintuitively, the expansion gap paradox, i.e., a dependence on the initial temperature of the effective relaxation time even close to equilibrium when heating. Here, we show that a distinguishable-particle lattice model can capture both the asymmetry and the paradox. We quantitatively characterize the energetic states of the particle configurations using a physical realization of the fictive temperature called the structural temperature, which, in the heating case, displays a strong spatial heterogeneity. The system relaxes by nucleation and expansion of warmer mobile domains having attained the final temperature, against cooler immobile domains maintained at the initial temperature. A small population of these cooler regions persists close to equilibrium, thus explaining the paradox.
\end{abstract}

DOI: 10.1103/PhysRevLett.124.095501

Kovacs' series of experiments [1] is fundamental to our present understanding of aging and memory properties in glassy materials [2-4]. In [1], the volume relaxation of polymer glasses has been analyzed by performing rapid temperature changes, or temperature jumps, focusing on experimental protocols implementing one or two successive temperature shifts.

On one hand, the double jump protocol illustrates Kovacs' effect evidencing memory properties in glasses. After a temperature down-jump, the polymer is annealed for an appropriate duration so that a further up-jump to a final temperature $T_{f}$ immediately takes the volume to its equilibrium value at $T_{f}$. Because of memory effects, the sample, however, is not yet at equilibrium and its volume indeed rises before dropping finally to equilibrium, i.e., the Kovacs hump. Kovacs' effect has been widely studied using empirical mean-field models including the ToolNarayanaswamy-Moynihan [5-7] and the Kovacs-AklonisHutchinson-Ramos [8] models. A temperature jump more precisely acts directly only on the phonon temperature. The memory is accounted for in these theories using a fictive temperature $T_{F}(t)$, which describes some internal state of the material with a dynamics generally lagging behind that of phonons [5].

On the other hand, single-jump experiments lead to the expansion gap paradox, also called the $\tau_{\text {eff }}$ paradox, which is a more puzzling phenomenon [9-16]. The effective relaxation rate $\tau_{\text {eff }}^{-1}$ of the polymeric system studied by
Kovacs, depends persistently on the initial temperature, and apparently, even arbitrarily close to equilibrium. In contrast, for many nonglassy systems, linearized dynamics applies at long times and yields the same $\tau_{\text {eff }}$ for both up- and downjumps. Such a strong material memory in glasses, however, cannot be reproduced by Tool-Narayanaswamy-Moynihan or Kovacs-Aklonis-Hutchinson-Ramos models, and has only been accounted for by their stochastic counterparts, namely the stochastic version of a free-volume model [17] and, more recently, the stochastic constitutive model [18]. The reasons for the failure of mean-field models and for the importance of stochastic fluctuations are not well understood in this case.

We reproduce Kovacs' expansion gap for the first time using a microscopic particle model, going beyond meanfield descriptions. Specifically, we adopt the distinguishable-particle lattice model (DPLM) [19]. The phonon temperature is modeled by the bath temperature of the kinetic Monte Carlo simulation. We observe an expansion gap in the system energy relaxation, analogous to enthalpy relaxation in experiments [20]. By studying spatial profiles of particle displacements and interactions, we provide an intuitive resolution of the paradox.

The DPLM displays several particle dynamics features characteristic of glasses and possesses exactly solvable equilibrium statistics [19]. A wide range of values of the fragility index can be obtained by varying the interaction energy distribution of the model [21]. Kovacs effect can 
also be reproduced [22]. The DPLM is defined on a regular square lattice with $N$ particles. At site $i$ a particle denoted by the label $s_{i}=1, \ldots, N$ may be located. For an empty site, i.e., occupied by a void, $s_{i}=0$. As a distinctive feature, the interaction energy of nearest neighboring particles $V_{i j s_{i} s_{j}}$ is both site dependent and particle dependent, as indicated by site indices $(i, j)$ and particle indices $\left(s_{1}, s_{2}\right)$ respectively. We write the system energy as

$$
E=\sum_{\langle i j\rangle^{\prime}} V_{i j s_{i} s_{j}}
$$

where the sum $\sum_{\langle i j\rangle^{\prime}}$ is restricted to occupied nearest neighboring sites. All possible interactions $V_{i j k l}$, defined for each lattice link $(i, j)$ and particle couple $(k, l)$, are drawn according to the a priori probability distribution $g(V)$. On the other hand, the interaction energies at equilibrium, i.e., the $V_{i j s_{i} s_{j}}$, have been proven [19] to be distributed as $p_{\text {eq }}(V, T)=g(V) e^{-V / k_{B} T} / \mathcal{N}(T)$, where $\mathcal{N}(T)$ is a normalization factor. In the following, we will use natural units with $k_{B}=1$. We take $g$ as a uniform distribution defined on the interval $V \in\left[V_{0}, V_{1}\right]$, with $V_{1}=$ $-V_{0}=0.5$ [19]. Hence, $p_{\text {eq }}$ yields a simple exponential dependence on the interaction energy. [See Supplemental Material (SM) [23] for details.]

We simulate the single-jump protocol [1] starting from equilibrium configurations [19] at some initial temperatures $T_{i}$. Then, the bath temperature $T$, representing the phonon temperature, is set instantaneously at time 0 to the final value $T_{f}$. We study the fractional deviation [1] $\delta_{E}(t)$ of the system energy $E(t)$ from its equilibrium value $E_{\infty}$ at $T_{f}$, i.e., $\quad \delta_{E}(t)=\left[E(t)-E_{\infty}\right] /\left|E_{\infty}\right| . \quad E(t)$ and $E_{\infty}<0$ are computed using Eq. (1) (see SM [23]). Figure 1(a) reports $\delta_{E}(t)$ for a set of symmetric temperature jumps, of similar relative magnitudes as in [1]. As shown in Fig. 1, $\delta_{E}$ from DPLM simulations closely resembles experimental results in [1] and correctly reproduces the up-down asymmetry of the approach to equilibrium. At time 0 the asymmetry is due to an equilibrium heat capacity decreasing with $T$ (see SM [23]). However, the much slower relaxation for the upjumps compared with the down-jumps, successfully reproduced here, is nontrivial and has been the focus of many studies [9-16].

Let us define the effective relaxation time $\tau_{\text {eff }}$ [1] by $\tau_{\text {eff }}^{-1}(t)=-\left[\delta_{E}(t)\right]^{-1} d \delta_{E}(t) / d t$, yielding a constant for an exponentially decaying $\delta_{E}(t)$. Results for $\tau_{\text {eff }}(t)$ against $\delta_{E}(t)$ are reported in Fig. 1(b) showing very similar features to those reported in [1]. Most importantly, we observe as in [1] that the data for $\tau_{\text {eff }}$ have not converged to a single limiting value independent of $T_{i}$, even close to equilibrium at $\left|\delta_{E}\right| \simeq 0$, creating the expansion gap paradox. The inset reports data close to equilibrium: Down-jump data show a clear convergence among themselves and with respect to the up-jump data at small jumps, whereas convergence is not observed for large up-jumps at the smallest $\delta_{E}$ studied.
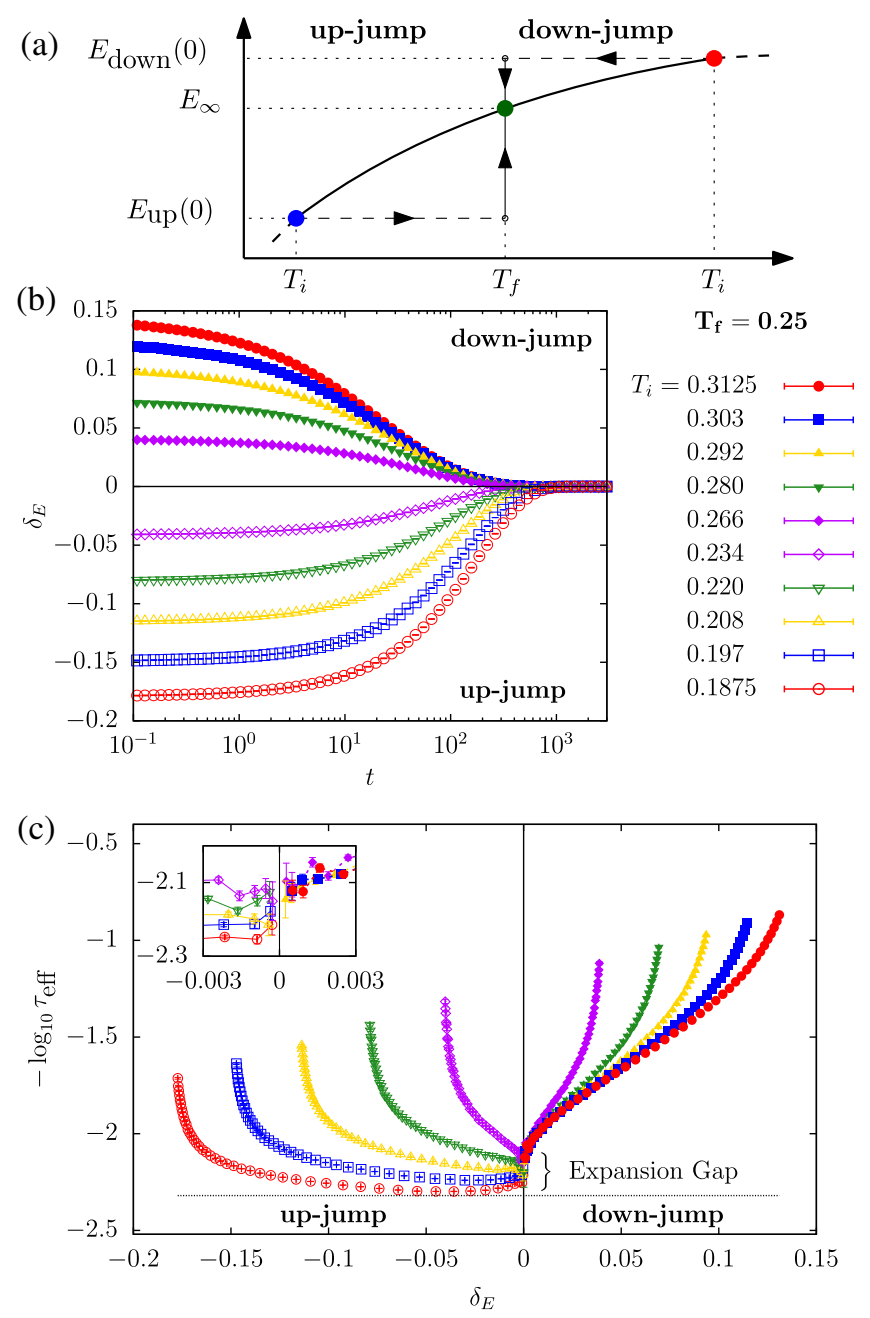

FIG. 1. (a) Schematic diagram for single-temperature jump protocol for equilibrium samples at initial temperature $T_{i}$ which are then cooled (for $T_{i}>T_{f}$, i.e., down-jump) or heated (for $T_{i}<T_{f}$, i.e., up-jump) to the final temperature $T_{f}$. (b) Data from DPLM simulations for $T_{f}=0.25$ and different values of $T_{i}$. The asymmetry of the approach between up- and down-jumps is observed. (c) Results on $\tau_{\text {eff }}$ measured using data in (b). Data close to equilibrium with $\left|\delta_{E}(t)\right| \leq 0.003$ are shown in the inset.

To the best of our knowledge, among the constitutive models $[5-8,17,18]$ only the stochastic free-volume model [17] and the stochastic constitutive model [18], accounting for dynamic heterogeneities, can reproduce the gap. Being able to qualitatively recover the most important experimental features by means of a microscopic particle model is clearly important for a deeper understanding of the aging dynamics.

Compared to constitutive models, an advantage of our approach is that it allows us to analyze the differences between up- and down-jump dynamics from the real-space perspective, going beyond mean-field descriptions. We define a local particle displacement $d(\vec{x}, t)$ and a local particle persistence, i.e., an overlap field, $\tilde{q}(\vec{x}, t)$ such that the average overlap $q(t)$, defined as $\tilde{q}(\vec{x}, t)$ averaged over 


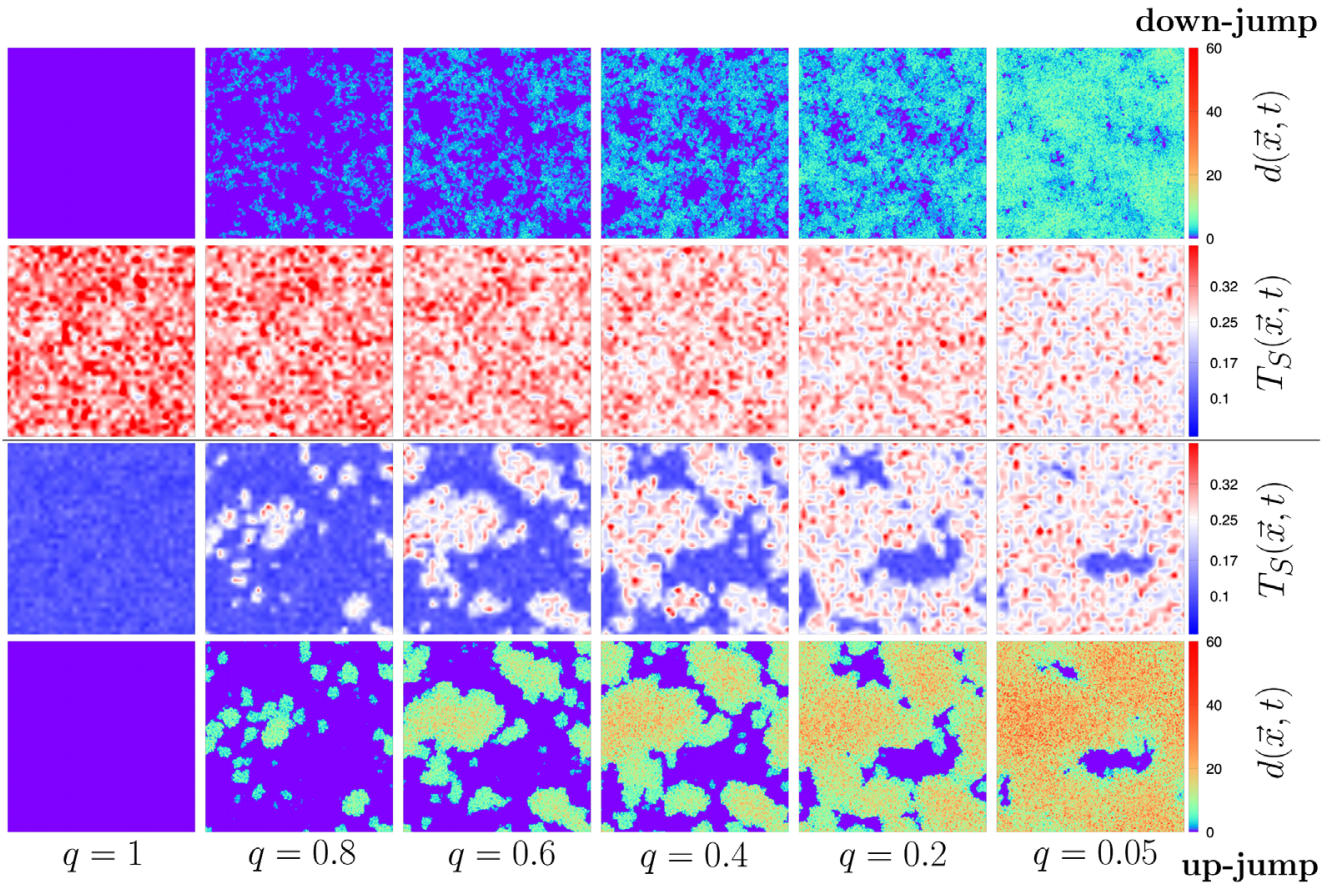

FIG. 2. Snapshots of structural temperature $T_{S}(\vec{x}, t)$ (second and third rows) and particle displacement $d(\vec{x}, t)$ (first and fourth rows) for down-jump from $T_{i}=0.3125$ (first and second rows) and up-jump from $T_{i}=0.1$ (third and fourth rows) to final temperature $T_{f}=0.25$ in a system of linear size $L=200$ with coarse-graining length scale $\ell=5$. Different columns refer to average overlap $q$ corresponding to increasing time, from left to right. $T_{S}(\vec{x}, t)$ and $d(\vec{x}, t)$ show homogeneous evolution for the down-jump, but strongly heterogeneous evolution with large domains for the up-jump. For the up-jump case immobile domains coincide with low structural temperature domains. See videos in SM [23] for full dynamics.

sites occupied at $t$, gives the fraction of particles still located at their original positions at time $t$ (see SM [23] for details). Figure 1 in the SM [23] displays $q(t)$ against $t$. In Fig. 2 we report the evolution of the local displacement $d(\vec{x}, t)$ for selected values of $q$, providing a useful measure of the progress of the relaxation. Large jump magnitudes make qualitative features of the dynamics more clearly observable. Figure 2 shows a much more heterogeneous growth of $d(\vec{x}, t)$ in the up-jump case. Well-separated domains with highly mobile particles nucleate and invade immobile domains. Hence, a strong spatial heterogeneity dominates the up-jump relaxation.

In order to understand the emergence of the heterogeneous up-jump dynamics, we study the energy states of the particle configurations by measuring the probability distribution $p(V)$ of the realized interactions $V_{i j s_{i} s_{j}}$. In Fig. 3(a) and (b) $p(V)$ is reported for different values of the average overlap $q$. Figure 3(a) shows that for down-jumps the evolution of $p(V)$ occurs uniformly for the whole range of $V$. Furthermore, $p(V)$ can be reasonably fitted by a single equilibrium distribution $p_{\mathrm{eq}}(V, T)$, with $T$ decreasing monotonically with $1-q$ and thus also with $t$. For the up-jump data reported in Fig. 3(b), there is a remarkable difference between the evolution of high- and low-energy interactions: For $q=0.8$, at $V \gtrsim 0.25$ the distribution has already attained the same slope in the semilog plot as the final equilibrium one at $T_{f}$, while values of $p(V)$ for low-energy interactions $V \lesssim-0.25$ are still very close to those of the initial temperature $T_{i}$. Indeed, $p(V)$ for a wide range of $q$ is very well fitted by a superposition of two equilibrium distributions

$$
p(V)=q p_{\mathrm{eq}}\left(V, T_{i}\right)+(1-q) p_{\mathrm{eq}}\left(V, T_{f}\right),
$$

suggesting that the particle configurations in the mobile regions, whose relative extent is $1-q$, have reached equilibrium at the final temperature $T_{f}$ while the immobile regions, whose extent is $q$, have interactions distributed according to $p_{\text {eq }}$ at the initial temperature $T_{i}$.

The results above suggest the existence of a strong spatial heterogeneity in the distribution of the realized particle pair-interactions only for the up-jump case. Further, we compute a temperature $T_{S}$ we call the structural temperature. In physical terms, $T_{S}$ measures how well particles are locally packed, i.e., a low temperature corresponds to a better bonded and more stable configuration. This definition can be applied to a wide range of materials and it should not be regarded as specific to the present case. For the DPLM, we define it as a local temperature $T_{S}(\vec{x}, t)$ at position $\vec{x}$ based on the interaction $\bar{V}_{\ell}(\vec{x}, t)$ averaged over a square domain of linear size $\ell$ centered at $\vec{x}$. Requiring that $T_{S}$ coincides with the bath temperature at equilibrium, $T_{S}$ is computed by solving numerically $\bar{V}_{\ell}(\vec{x}, t)=$ $\int d V V p_{\text {eq }}\left(V, T_{S}\right)$. Note that $T_{S}$ is analogous to Tool's 

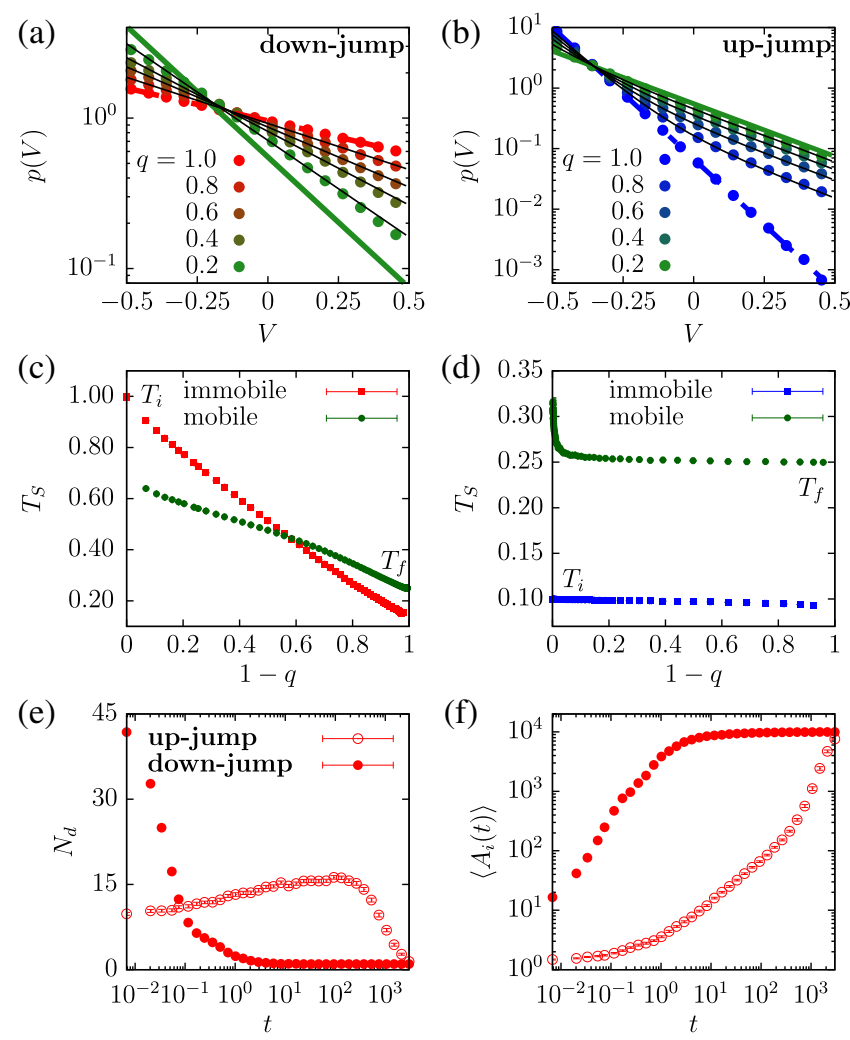

FIG. 3. (a) and (b) Semilog plot of $p(V)$ in dots, at different overlap $q$, for the down- $\left(T_{i}=1.0\right)$ and the up- $\left(T_{i}=0.1\right)$ jumps with common $T_{f}=0.25$. The final equilibrium distribution is drawn in solid lines while the initial ones in dashed lines. (a) Down-jump data superposed to single-temperature fits in black lines. (b) Up-jump data superposed to Eq. (2). In both cases fits show good agreement. (c) and (d) average $T_{S}$ for mobile and immobile domains against $1-q$, which increases with time. (c) (Down-jump), $T_{S}$ in both regions steadily decreases. (d) (Upjump) $T_{S}$ in mobile regions converges fast to $T_{f}$ while being close to $T_{i}$ in immobile regions. (e) Number of mobile domains $N_{d}$ versus time. (f) Average domain area.

fictive temperature [5], local values of which have also been studied before [24,25].

Figure 2 shows the evolution of $T_{S}(\vec{x}, t)$ for $\ell=5$. Similar to the displacement $d(\vec{x}, t)$, we see that the evolution of $T_{S}(\vec{x}, t)$ is spatially homogeneous for the down-jump. For the up-jump, high- $T_{S}$ domains with $T_{S} \simeq$ $T_{f}$ develop in spatial correspondence with the high mobility regions. Whereas, the immobile regions maintain the initial temperature, i.e., $T_{S} \simeq T_{i}$. Moreover, some low- $T_{S}$ immobile domains remain even at the very late stage of relaxation at $q=0.05$, thus constituting a remnant of the initial temperature acting on the dynamics, although equilibrium is already reached almost everywhere else. Figures 3(c) and 3(d) report the evolution of the average $T_{S}$ for mobile and immobile regions as a function of $1-q$ which increases with time. For the up-jump case shown in Fig. 3(d), $T_{S}$ quickly converges to $T_{f}$ in the mobile

domains while it remains close to $T_{i}$ in the immobile regions. All these results are fully consistent with the good fits to $p(V)$ in Fig. 3(b) using Eq. (2). Whereas, Fig. 3(c) shows that, in the down-jump case, $T_{S}$ steadily evolves in both regions taking on relatively similar values. Hence $P(V, t)$ at each time can be described approximately by a single $p_{\text {eq }}$ as reported in Fig. 3(a).

The structural temperature heterogeneity observed for the up-jumps can be understood in terms of a stability argument of propagating fronts as discussed in the SM [23]. We argue that the strong spatial fluctuations are caused by a spatial instability due to the autocatalytic nature of the heating of glasses. In contrast, for a temperature downjump, the autoretarding nature of cooling leads to a stable and thus homogeneous evolution of the structural temperature, resulting in weak memory effects and converging relaxation rates.

We further characterize the up-jump dynamics by measuring the number of mobile domains $N_{d}$ and their average area $\left\langle A_{i}(t)\right\rangle$ (see SM [23]). Results are reported in Figs. 3(e) and 3(f). For the down-jump case, the initial value $N_{d} \simeq$ $N_{v}=50$ indicates that mobile domains are independently generated with small energy barriers by individual voids and rapidly coalesce, inducing the drop in $N_{d}$. For the upjump case, $N_{d}$ starts from a smaller initial value and has a logarithmic growth due to nucleation events with significant energy barriers until coalescence causes their number to decrease. Finally, we notice that the average domain area, reported in Fig. 3(f), displays a much slower growth in the up-jump case because of the slow domain motions explained above.

Interestingly, the aging dynamics described in this work shares important features with the melting of ultrastable glasses [26] for which the front propagation corresponds to high- $T_{S}$ domains nucleated at the free surface invading the inner region originally at lower $T_{S}$. Furthermore, it would be worthwhile to generalize this present work to more complex thermal treatments and reproduce dual relaxation times reported previously $[25,27]$.

The structural temperature introduced in this work is, to the best of our knowledge, the first example of a physical realization of Tool's fictive temperature [5]. As an advancement, it is measurable from particle simulations based on well-defined microscopic dynamics, in contrast to the fictive temperature which follows separate empirical evolution rules [4]. Our results stress the importance of its spatial heterogeneity in understanding the expansion gap paradox. This naturally explains why mean-field models with a global fictive temperature [5-8] in general have difficulty reproducing the paradox. It also justifies the stochastic models $[17,18]$ in which different stochastic realizations empirically represent local regions at different stages of evolution. We believe that the structural temperature will be of general importance in the study of nonequilibrium behaviors of glasses. It should be of interest to 
measure it experimentally by means of, for example, electron correlation microscopy [28].

In summary, Kovacs' expansion gap paradox in energy relaxation is reproduced based on kinetic Monte Carlo simulations of a particle model in two dimensions. A structural temperature is introduced to characterize the energy states of the particle configurations. After an upjump of the bath temperature from $T_{i}$ to $T_{f}$, a large spatial heterogeneity is observed in both local particle displacement and local structural temperature. The evolution of the latter is characterized by the nucleation and coarsening of $T_{f}$ domains invading the original $T_{i}$ domains. Relaxation dynamics persistently depend on $T_{i}$ because isolated $T_{i}$ domains survive even close to the end of the relaxation. This leads to strong memory effects and explains the paradox.

Kovacs' experiments are important because exceptional material properties often provide the deepest insights. Overcoming the long-standing challenge of reproducing the expansion gap using a microscopic particle model, our results do not only provide a possible intuitive understanding of the paradox, but also support the validity of the DPLM as a reliable tool for studying glassy dynamics. Finally, the equilibrium dynamics of the DPLM has been described theoretically $[29,30]$. It will be interesting to extend the analysis to the present far from equilibrium situations.

We gratefully acknowledge Haihui Ruan and Giorgio Parisi for interesting discussion and comments. We are thankful for the support of Hong Kong GRF (Grant No. 15330516) and PolyU (Grants No. 1-ZVGH and No. G-UAF7), and National Natural Science Foundation of China (Grants No. 11974297, No. 91741101, and No. 91752204), and Science and Technology Innovation Committee Foundation of Shenzhen Grant No. JCYJ20170817105533245.

*C.H.Lam@ @olyu.edu.hk

[1] A. J. Kovacs, in Fortschritte Der HochpolymerenForschung (Springer, Berlin, Heidelberg, 1964), pp. 394507, https://doi.org/10.1007/BFb0050366.

[2] Polymer Glasses, edited by C. B. Roth (CRC Press, Boca Raton, 2016), p. 572, https://doi.org/10.4324/ 9781315305158.

[3] C. A. Angell, K. L. Ngai, G. B. McKenna, P. F. McMillan, and S. W. Martin, J. Appl. Phys. 88, 3113 (2000).

[4] I. M. Hodge, J. Non-Cryst. Solids 169, 211 (1994).

[5] A. Q. Tool, J. Am. Ceram. Soc. 29, 240 (1946).
[6] O. S. Narayanaswamy, J. Am. Ceram. Soc. 54, 491 (1971).

[7] C. T. Moynihan, P. B. Macedo, C. J. Montrose, C. J. Montrose, P. K. Gupta, M. A. DeBolt, J.F. Dill, B.E. Dom, P. W. Drake, A. J. Easteal, P. B. Elterman, R. P. Moeller, H. Sasabe, and J. A. Wilder, Ann. N.Y. Acad. Sci. 279, 15 (1976).

[8] A. J. Kovacs, J. J. Aklonis, J. M. Hutchinson, and A. R. Ramos, J. Polym. Sci. 17, 1097 (1979).

[9] G. B. McKenna, Y. Leterrier, and C. R. Schultheisz, Polym. Eng. Sci. 35, 403 (1995).

[10] G. B. McKenna, M. G. Vangel, A. L. Rukhin, S. D. Leigh, B. Lotz, and C. Straupe, Polymer 40, 5183 (1999).

[11] S. Kolla and S. L. Simon, Polymer 46, 733 (2005).

[12] T. Hecksher, N. B. Olsen, K. Niss, and J. C. Dyre, J. Chem. Phys. 133, 174514 (2010).

[13] T. Hecksher, N. B. Olsen, and J. C. Dyre, J. Chem. Phys. 142, 241103 (2015).

[14] S. Banik and G. B. McKenna, Phys. Rev. E 97, 062601 (2018).

[15] L. C. Struik, Polymer 38, 4677 (1997).

[16] L. C. Struik, Polymer 38, 5233 (1997).

[17] R. E. Robertson, R. Simha, and J. G. Curro, Macromolecules 17, 911 (1984).

[18] G. A. Medvedev and J. M. Caruthers, Macromolecules 48, 788 (2015).

[19] L.-H. Zhang and C.-H. Lam, Phys. Rev. B 95, 184202 (2017).

[20] S. Montserrat, J. Polym. Sci. B 32, 509 (1994).

[21] C.-S. Lee, M. Lulli, L.-H. Zhang, H.-Y. Deng, and C.-H. Lam,arXiv:1909.03240.

[22] M. Lulli, L.-H. Zhang, C.-S. Lee, H.-Y. Deng, and C.-H. Lam, arXiv:1910.10374.

[23] See Supplemental Material at http://link.aps.org/ supplemental/10.1103/PhysRevLett.124.095501 for details about the DPLM definition, analytical results, discussion of the up-jump dynamic stability, details on the domain detection and on overlap and voids dynamics, also displayed in two (up- and down-jump) videos where voids are marked by black dots.

[24] A. S. Keys, J. P. Garrahan, and D. Chandler, Proc. Natl. Acad. Sci. U.S.A. 110, 4482 (2013).

[25] A. Wisitsorasak and P. G. Wolynes, J. Phys. Chem. B 118, 7835 (2014).

[26] C. Rodríguez-Tinoco, M. Gonzalez-Silveira, J. Ràfols-Ribé, A. Vila-Costa, J.C. Martinez-Garcia, and J. RodríguezViejo, Phys. Rev. Lett. 123, 155501 (2019).

[27] D. Cangialosi, V. M. Boucher, A. Alegría, and J. Colmenero, Phys. Rev. Lett. 111, 095701 (2013).

[28] P. Zhang, J. J. Maldonis, Z. Liu, J. Schroers, and P. M. Voyles, Nat. Commun. 9, 1129 (2018).

[29] C.-H. Lam, J. Stat. Mech. (2018) 023301.

[30] H.-Y. Deng, C.-S. Lee, M. Lulli, L.-H. Zhang, and C.-H. Lam, J. Stat. Mech. (2019) 094014. 